\title{
Time-Dependent Ginzburg-Landau Theory and Duality
}

\author{
Adriaan M. J. Schakel \\ Institut für Theoretische Physik \\ Freie Universität Berlin \\ Arnimallee 14, 14195 Berlin
}

\begin{abstract}
In the first part of this review paper, the time-dependent Ginzburg-Landau theory is derived starting from the microscopic BCS model with the help of a derivative expansion. Special attention is paid to two space dimensions, where the entire crossover from the weak-coupling BCS limit to the strong-coupling BEC limit of tightly bound fermion pairs is accessible analytically. The second part deals with the dual approach to the time-independent Ginzburg-Landau theory in three space dimensions. In this approach, the magnetic vortices of a superconductor play the central role, and the superconductor-to-normal phase transition is understood as a proliferation of these vortices.
\end{abstract}

\section{INTRODUCTION}

The role of topological defects in symmetry-breaking phase transitions cannot be underestimated. The prime example of a defect-driven (equilibrium) transition is the superfluid-to-normal phase transition in a ${ }^{4} \mathrm{He}$ film, which can be understood as the unbinding of vortex-antivortex pairs [1].2]. Since the very existence of an ordered state and the presence of topological defects are both manifestations of spontaneously broken symmetries, it doesn't come as a surprise that various phase transitions can be understand in terms of defects. To appreciate this point of view, note that defects are regions, usually macroscopic in size, where the symmetry is broken differently from that in the rest of the system. Often, the symmetry is completely restored in the defect cores, meaning that they are in the normal state. (In systems like superfluid ${ }^{3} \mathrm{He}$ with a large symmetry group, the defect cores may be ordered states themselves, but of a different character.) Now, when defects with a normal-state core proliferate, the ordered state gets disordered and converted to the normal state.

In recent years also the dynamics of phase transitions and the accompanying formation of defects have received much attention (see Ref. [3] for reviews). An important role is played here by the so-called time-dependent Ginzburg-Landau theory, which provides a phenomenological approach. Ideally, one would like to start with a microscopic model of the system under consideration and derive the time-dependent Ginzburg-Landau theory by integrating out irrelevant degrees of freedom, thus turning the phenomenological theory into an effective one. For a superconductor this program, at least in the time-independent case, has been carried out by Gorkov [4]. Starting from the microscopic theory of Bardeen, Cooper, and Schrieffer (BCS) [5], he derived by means of a Green function method the Ginzburg-Landau theory of superconductivity which had been proposed as a phenomenological theory seven years before the BCS theory was formulated [6].

In the first part of this review paper, we reformulate Gorkov's derivation (extended to include time dependence) in terms of the functional-integral approach to quantum field theory. We will not only study the weak-coupling BCS limit of loosely bound Cooper pairs, but consider arbitrary values of the coupling constant $\lambda_{0}$, including the limit $\lambda_{0} \rightarrow-\infty$, where the fermions form tightly bound pairs. When going from the weak-coupling to the large- $\lambda_{0}$ limit, the dynamics changes from dissipative to non-dissipative behavior.

In the second part of this review paper, we discuss the dual approach to the time-independent Ginzburg-Landau theory. It is a formulation directly in terms of magnetic vortices, in which the superconductor-to-normal is described as a proliferation of vortices.

\section{A. Notation}

We adopt Feynman's notation and denote a spacetime point by $x=x_{\mu}=(t, \mathbf{x}), \mu=0,1, \cdots, d$, with $d$ the number of space dimensions, while the energy $k_{0}$ and momentum $\mathbf{k}$ will be denoted by $k=k_{\mu}=\left(k_{0}, \mathbf{k}\right)$. The time derivative $\partial_{0}=\partial / \partial t$ and the gradient $\nabla$ are sometimes combined in a single vector $\tilde{\partial}_{\mu}=\left(\partial_{0},-\nabla\right)$. The tilde on $\partial_{\mu}$ is to alert the reader for the minus sign appearing in the spatial components of this vector. We define the scalar product $k \cdot x=k_{\mu} x_{\mu}=k_{\mu} g_{\mu \nu} k_{\nu}=k_{0} t-\mathbf{k} \cdot \mathbf{x}$, with $g_{\mu \nu}=\operatorname{diag}(1,-1, \cdots,-1)$ and use Einstein's summation convention. Because of the minus sign in the definition of the vector $\tilde{\partial}_{\mu}$, it follows that $\tilde{\partial}_{\mu} a_{\mu}=\partial_{0} a_{0}+\nabla \cdot \mathbf{a}$, with $a_{\mu}$ an arbitrary vector.

Integrals over spacetime are denoted by

$$
\int_{x}=\int_{t, \mathbf{x}}=\int \mathrm{d} t \mathrm{~d}^{d} x,
$$


while those over energy and momentum by

$$
\int_{k}=\int_{k_{0}, \mathbf{k}}=\int \frac{\mathrm{d} k_{0}}{2 \pi} \frac{\mathrm{d}^{d} k}{(2 \pi)^{d}} .
$$

When no integration limits are indicated, the integrals are assumed to be over all possible values of the integration variables.

Natural units $\hbar=k_{\mathrm{B}}=1$ are adopted throughout.

\section{TIME-DEPENDENT GINZBURG-LANDAU THEORY}

In this section, we derive the time-dependent Ginzburg-Landau theory for a superconductor, starting from the microscopic BCS model. Following Ref. [7], we integrate out the fermionic degrees of freedom in favor of a bosonic field describing the fermion pairs and use a derivative expansion method. We wish to obtain the Ginzburg-Landau theory not only in the weakcoupling BCS limit of loosely bound and overlapping Cooper pairs, but for arbitrary values of the coupling constant, in particular in the limit $\lambda_{0} \rightarrow-\infty$ where the fermions form tightly bound pairs [8, 9]. This crossover between the two limits has recently been studied in detail [10-13]. Since in two space dimensions this region becomes accessible by analytical methods, we shall, unless stated otherwise, restrict ourselves to $d=2$ when carrying out momentum integrals.

\section{A. BCS Model}

The BCS Lagrangian reads [14]

$$
\begin{aligned}
\mathcal{L} & =\psi_{\uparrow}^{*}\left[i \partial_{0}-\xi(-i \nabla)\right] \psi_{\uparrow}+\psi_{\downarrow}^{*}\left[i \partial_{0}-\xi(-i \nabla)\right] \psi_{\downarrow}-\lambda_{0} \psi_{\uparrow}^{*} \psi_{\downarrow}^{*} \psi_{\downarrow} \psi_{\uparrow} \\
& =: \mathcal{L}_{0}+\mathcal{L}_{\mathrm{i}}
\end{aligned}
$$

where $\mathcal{L}_{\mathrm{i}}=-\lambda_{0} \psi_{\uparrow}^{*} \psi_{\downarrow}^{*} \psi_{\downarrow} \psi_{\uparrow}$ is a contact interaction term, representing the effective attraction between electrons with bare coupling constant $\lambda_{0}<0$, and $\mathcal{L}_{0}$ is the remainder. In Eq. (1), the field $\psi_{\uparrow(\downarrow)}$ is an anticommuting field describing the electrons with mass $m$ and spin up (down); $\xi(-i \nabla)=\epsilon(-i \nabla)-\mu_{0}$, with $\epsilon(-i \nabla)=-\nabla^{2} / 2 m$, is the kinetic energy operator with the bare chemical potential $\mu_{0}$ subtracted.

For computational convenience we introduce Nambu's notation and rewrite the Lagrangian (1) in terms of a two-component field

$$
\psi=\left(\begin{array}{c}
\psi_{\uparrow} \\
\psi_{\downarrow}^{*}
\end{array}\right) \quad \psi^{\dagger}=\left(\psi_{\uparrow}^{*}, \psi_{\downarrow}\right) .
$$

In order to integrate out the fermionic degrees of freedom, the zero-temperature partition function represented as a functional integral

$$
Z=\int \mathrm{D} \psi^{\dagger} \mathrm{D} \psi \exp \left(i \int_{x} \mathcal{L}\right)
$$

must be written in a form bilinear in the electron fields. This is achieved by rewriting the quartic interaction term as a functional integral over auxiliary fields $\Delta$ and $\Delta^{*}$ :

$$
\begin{aligned}
& \exp \left(-i \lambda_{0} \int_{x} \psi_{\uparrow}^{*} \psi_{\downarrow}^{*} \psi_{\downarrow} \psi_{\uparrow}\right)= \\
& \int \mathrm{D} \Delta^{*} \mathrm{D} \Delta \exp \left[-i \int_{x}\left(\Delta^{*} \psi_{\downarrow} \psi_{\uparrow}+\psi_{\uparrow}^{*} \psi_{\downarrow}^{*} \Delta-\frac{1}{\lambda_{0}}|\Delta|^{2}\right)\right] .
\end{aligned}
$$

The field equation for $\Delta^{*}$,

$$
\Delta=\lambda_{0} \psi_{\downarrow} \psi_{\uparrow}
$$

shows that the auxiliary field describes electron pairs. We will therefore refer to it as pair field. The partition function thus becomes 


$$
\begin{aligned}
& Z=\int \mathrm{D} \psi^{\dagger} \mathrm{D} \psi \int \mathrm{D} \Delta^{*} \mathrm{D} \Delta \exp \left(\frac{i}{\lambda_{0}} \int_{x}|\Delta|^{2}\right) \\
& \times \exp \left[i \int_{x} \psi^{\dagger}\left(\begin{array}{cc}
i \partial_{0}-\xi(-i \nabla) & -\Delta \\
-\Delta^{*} & i \partial_{0}+\xi(-i \nabla)
\end{array}\right) \psi\right] .
\end{aligned}
$$

Changing the order of integration and performing the Gaussian integral over the Grassmann fields, we obtain

$$
Z=\int \mathrm{D} \Delta^{*} \mathrm{D} \Delta \exp \left(i S_{\mathrm{eff}}\left[\Delta^{*}, \Delta\right]+\frac{i}{\lambda_{0}} \int_{x}|\Delta|^{2}\right),
$$

with $S_{\text {eff }}$ the one-loop effective action which, using the identity $\operatorname{Det}(A)=\exp [\operatorname{Tr} \ln (A)]$, can be rewritten as

$$
S_{\text {eff }}\left[\Delta^{*}, \Delta\right]=-i \operatorname{Tr} \ln \left(\begin{array}{cc}
p_{0}-\xi(\mathbf{p}) & -\Delta \\
-\Delta^{*} & p_{0}+\xi(\mathbf{p})
\end{array}\right),
$$

where $p_{0}=i \partial_{0}$ and $\xi(\mathbf{p})=\epsilon(\mathbf{p})-\mu_{0}$, with $\epsilon(\mathbf{p})=\mathbf{p}^{2} / 2 m$.

The trace Tr appearing here needs some explanation. Explicitly, it is defined as

$$
S_{\mathrm{eff}}=-i \operatorname{Tr} \ln [K(p, x)]=-i \operatorname{tr} \ln \left[\left.K(p, x) \delta(x-y)\right|_{y=x}\right],
$$

where the trace $\operatorname{tr}$ is the usual one over discrete indices. We abbreviated the matrix appearing in (8) by $K(p, x)$ so as to cover the entire class of actions of the form

$$
S=\int_{x} \psi^{\dagger}(x) K(p, x) \psi(x) .
$$

The delta function in (9) arises because $K(p, x)$ is obtained as a second functional derivative of the action

$$
\frac{\delta^{2} S}{\delta \psi^{\dagger}(x) \delta \psi(x)}=\left.K(p, x) \delta(x-y)\right|_{y=x},
$$

each of which gives a delta function. Since the action has only one integral $\int_{x}$ over spacetime, one delta function remains. Because it is diagonal, it may be taken out of the logarithm and the effective action (9) can be written as

$$
\begin{aligned}
S_{\text {eff }} & =-\left.i \operatorname{tr} \int_{x} \ln [K(p, x)] \delta(x-y)\right|_{y=x} \\
& =-i \operatorname{tr} \int_{x} \int_{k} \mathrm{e}^{i k \cdot x} \ln [K(p, x)] \mathrm{e}^{-i k \cdot x} .
\end{aligned}
$$

In the last step, we used the integral representation of the delta function:

$$
\delta(x)=\int_{k} \mathrm{e}^{-i k \cdot x},
$$

shifted the exponential function $\exp (i k \cdot y)$ to the left, which is justified because the derivative $p_{\mu}$ does not operate on it, and, finally, set $y_{\mu}$ equal to $x_{\mu}$. We thus see that the trace $\operatorname{Tr}$ in (9) stands for the trace over discrete indices as well as the integration over spacetime and over energy and momentum. The integral $\int_{k}$ arises because the effective action (96) is a one-loop result with $k_{\mu}$ the loop energy and momentum.

In the mean-field approximation, the functional integral (7) is approximated by the saddle point:

$$
Z=\exp \left(i S_{\mathrm{eff}}\left[\Delta_{\mathrm{mf}}^{*}, \Delta_{\mathrm{mf}}\right]+\frac{i}{\lambda_{0}} \int_{x}\left|\Delta_{\mathrm{mf}}\right|^{2}\right),
$$

where $\Delta_{\mathrm{mf}}$ is the solution of the mean-field equation

$$
\frac{\delta S_{\mathrm{eff}}}{\delta \Delta^{*}(x)}=-\frac{1}{\lambda_{0}} \Delta(x) .
$$

If we assume the system to be spacetime independent, so that $\Delta_{\mathrm{mf}}(x)=\bar{\Delta}$, Eq. (15) yields the celebrated BCS gap equation [5]: 


$$
\begin{aligned}
\frac{1}{\lambda_{0}} & =-i \int_{k} \frac{1}{k_{0}^{2}-E^{2}(k)+i \eta} \\
& =-\frac{1}{2} \int_{\mathbf{k}} \frac{1}{E(\mathbf{k})},
\end{aligned}
$$

where $\eta$ is an infinitesimal positive constant that is to be set to zero at the end of the calculation, and

$$
E(\mathbf{k})=\sqrt{\xi^{2}(\mathbf{k})+|\bar{\Delta}|^{2}}
$$

is the spectrum of the elementary fermionic excitations.

\section{B. BCS to BEC}

For a constant pair field, the effective action can be calculated in closed form. Writing

$$
\left(\begin{array}{cc}
k_{0}-\xi(\mathbf{k}) & -\bar{\Delta} \\
-\bar{\Delta}^{*} & k_{0}+\xi(\mathbf{k})
\end{array}\right)=\left(\begin{array}{cc}
k_{0}-\xi(\mathbf{k}) & 0 \\
0 & k_{0}+\xi(\mathbf{k})
\end{array}\right)-\left(\begin{array}{cc}
0 & \bar{\Delta} \\
\bar{\Delta}^{*} & 0
\end{array}\right)
$$

and expanding the second logarithm in a Taylor series, we recognize the form

$$
\begin{aligned}
& S_{\text {eff }}\left[\bar{\Delta}^{*}, \bar{\Delta}\right]= \\
& -i \operatorname{Tr} \ln \left(\begin{array}{cc}
k_{0}-\xi(\mathbf{k}) & 0 \\
0 & k_{0}+\xi(\mathbf{k})
\end{array}\right)-i \operatorname{Tr} \ln \left(1-\frac{|\bar{\Delta}|^{2}}{k_{0}^{2}-\xi^{2}(\mathbf{k})}\right),
\end{aligned}
$$

where we ignored an irrelevant constant. The integral over the loop energy $k_{0}$ can be carried out to yield for the effective Lagrangian

$$
\mathcal{L}_{\text {eff }}=\int_{\mathbf{k}}[E(\mathbf{k})-\xi(\mathbf{k})]
$$

To this one-loop result we have to add the tree term $|\bar{\Delta}|^{2} / \lambda_{0}$. Expanding $E(\mathbf{k})$ in a Taylor series, we see that the effective Lagrangian also contains a term quadratic in $\bar{\Delta}$. This term diverges in the ultraviolet. To render the theory finite to this order, we have to introduce a renormalized coupling constant $\lambda$ defined by:

$$
\frac{1}{\lambda}=\frac{1}{\lambda_{0}}+\frac{1}{2} \int_{\mathbf{k}} \frac{1}{|\xi(\mathbf{k})|}
$$

To this order in the loop expansion there is no renormalization of the chemical potential, so that we can write $\mu=\mu_{0}$. We regularize the diverging integral in Eq. (21) by introducing a momentum cutoff $\Lambda$. In, for example, $d=3$, we then obtain

$$
\frac{1}{\lambda}=\frac{1}{\lambda_{0}}+\frac{m}{2 \pi^{2}} \Lambda
$$

where we omitted the (irrelevant) finite part of the integral. It should be remembered that the bare coupling constant $\lambda_{0}$ is negative, so that the interaction between the fermions is attractive. We can distinguish two limits. One, the famous weakcoupling BCS limit, which is obtained by taking the bare coupling constant to zero, $\lambda_{0} \rightarrow 0^{-}$. Second, the limit which is obtained by letting $\lambda_{0} \rightarrow-\infty$. In this limit, the two-particle interaction is such that the fermions form tightly bound pairs of mass $2 m$ [8, 9].

To explicate this so-called Bose-Einstein condensation (BEC) limit in $d=2$, we swap the bare coupling constant for a more convenient parameter, namely the binding energy $\epsilon_{a}$ of a fermion pair in vacuum [15]. Both parameters characterize the strength of the contact interaction. To see the connection between the two, let us consider the Schrödinger equation for the problem at hand. In reduced coordinates, it reads

$$
\left[-\frac{\nabla^{2}}{m}+\lambda_{0} \delta(\mathbf{x})\right] \psi(\mathbf{x})=-\epsilon_{a} \psi(\mathbf{x}),
$$

where the reduced mass is $m / 2$ and the delta-function potential, with $\lambda_{0}<0$, represents the attractive contact interaction $\mathcal{L}_{\mathrm{i}}$ in the BCS Lagrangian (11). We stress that this is a two-particle problem in vacuum; it is not the famous Cooper problem of two 
interacting fermions on top of a filled Fermi sea. The equation is most easily solved by Fourier transforming it. This yields the bound-state equation

$$
\psi(\mathbf{k})=-\frac{\lambda_{0}}{\mathbf{k}^{2} / m+\epsilon_{a}} \psi(0),
$$

or

$$
-\frac{1}{\lambda_{0}}=\int_{\mathbf{k}} \frac{1}{\mathbf{k}^{2} / m+\epsilon_{a}}=\frac{1}{2} \nu(0) \ln \left(\frac{2 \epsilon_{\Lambda}}{\epsilon_{a}}\right),
$$

where $\nu(0)=m / 2 \pi$ is the two-dimensional density of states (per spin degree of freedom), and $\epsilon_{\Lambda}=\Lambda^{2} / 2 m$. This equation allows us to replace the bare coupling constant $\lambda_{0}$ with the binding energy $\epsilon_{a}$. When substituted in the gap equation Eq. (16), the latter becomes

$$
\int_{\mathbf{k}} \frac{1}{\mathbf{k}^{2} / m+\epsilon_{a}}=\frac{1}{2} \int_{\mathbf{k}} \frac{1}{E(\mathbf{k})} .
$$

By inspection, it is easily seen that this equation has a solution [9]

$$
\bar{\Delta} \rightarrow 0, \quad \mu_{0} \rightarrow-\frac{1}{2} \epsilon_{a}
$$

with a negative chemical potential. This is the strong-coupling BEC limit. To appreciate the physical significance of the specific value found for the chemical potential in this limit, we note that the spectrum $E_{\mathrm{b}}(\mathbf{q})$ of the two-fermion bound state measured relative to the pair chemical potential $2 \mu_{0}$ reads

$$
E_{\mathrm{b}}(\mathbf{q})=-\epsilon_{a}+\frac{\mathbf{q}^{2}}{4 m}-2 \mu_{0} .
$$

The negative value for $\mu_{0}$ found in (27) is precisely the condition for a Bose-Einstein condensation of the composite bosons in the $\mathbf{q}=0$ state-whence the name BEC limit.

Since there are two unknowns contained in the theory, viz. $\bar{\Delta}$ and $\mu$, a second equation is needed to determine these variables in the mean-field approximation [9]. It is provided by the requirement that the average fermion number $N$, which is obtained by differentiating the effective action (8) with respect to $\mu$

$$
N=\frac{\partial S_{\mathrm{eff}}}{\partial \mu}
$$

be fixed. If the system is spacetime independent, this reduces to

$$
\bar{n}=-i \operatorname{tr} \int_{k} G(k) \tau_{3},
$$

where $\bar{n}=N / V$, with $V$ the volume of the system, is the constant fermion number density, $\tau_{3}$ is the diagonal Pauli matrix in Nambu space,

$$
\tau_{3}=\left(\begin{array}{rr}
1 & 0 \\
0 & -1
\end{array}\right)
$$

and $G(k)$ is the Green function,

$$
\begin{aligned}
G(k) & =\left(\begin{array}{cc}
k_{0}-\xi(\mathbf{k}) & -\bar{\Delta} \\
-\bar{\Delta}^{*} & k_{0}+\xi(\mathbf{k})
\end{array}\right)^{-1} \\
& =\frac{1}{k_{0}^{2}-E^{2}(\mathbf{k})+i \eta}\left(\begin{array}{cc}
k_{0} \mathrm{e}^{i k_{0} \eta}+\xi(\mathbf{k}) & \bar{\Delta} \\
\bar{\Delta}^{*} & k_{0} \mathrm{e}^{-i k_{0} \eta}-\xi(\mathbf{k})
\end{array}\right) .
\end{aligned}
$$

Here, $\eta$ is an infinitesimal positive constant that is to be set to zero at the end of the calculation. The exponential functions in the diagonal elements of the Green function are an additional convergence factor needed in nonrelativistic theories [16]. If the integral over the loop energy $k_{0}$ in the particle number equation 30 is carried out, it takes the familiar form

$$
\bar{n}=\int_{\mathbf{k}}\left(1-\frac{\xi(\mathbf{k})}{E(\mathbf{k})}\right)
$$

The two equations $(16)$ and $(30)$ determine $\bar{\Delta}$ and $\mu$. 


\section{Derivative Expansion}

When the pair field $\Delta$ is spacetime-dependent, the integrals in $(8)$ cannot be evaluated in closed form because the logarithm contains energy-momentum operators and spacetime-dependent functions in a mixed order. To disentangle the integrals we have to resort to a derivative expansion [17] in which the logarithm is expanded in a Taylor series. Each term contains powers of the energy-momentum operator $p_{\mu}$ which acts on every spacetime-dependent function to its right. All these operators are shifted to the left by repeatedly applying the identity

$$
f(x) p_{\mu} g(x)=\left(p_{\mu}-i \tilde{\partial}_{\mu}\right) f(x) g(x)
$$

where $f(x)$ and $g(x)$ are arbitrary functions of spacetime and the derivative $\tilde{\partial}_{\mu}=\left(\partial_{0},-\nabla\right)$ acts only on the next object to the right. One then integrates by parts, so that all the $p_{\mu}$ 's act to the left where only a factor $\exp (i k \cdot x)$ stands. Ignoring total derivatives and taking into account the minus signs that arise when integrating by parts, one sees that all occurrences of $p_{\mu}$ (an operator) are replaced with $k_{\mu}$ (an integration variable). The exponential function $\exp (-i k \cdot x)$ can at this stage be moved to the left where it is annihilated by the function $\exp (i k \cdot x)$. The energy-momentum integration can now in principle be carried out and the effective action be cast in the form of an integral over a local density $\mathcal{L}_{\text {eff }}$ :

$$
S_{\mathrm{eff}}=\int_{x} \mathcal{L}_{\mathrm{eff}}
$$

This is in a nutshell how the derivative expansion works [17].

To apply it to the BCS model and derive the Ginzburg-Landau theory we use the following decomposition in (8):

$$
\left(\begin{array}{cc}
p_{0}-\xi(\mathbf{p}) & -\Delta \\
-\Delta^{*} & p_{0}+\xi(\mathbf{p})
\end{array}\right)=G_{0}^{-1}\left[1-G_{0}\left(\begin{array}{cc}
0 & \Delta \\
\Delta^{*} & 0
\end{array}\right)\right],
$$

where $G_{0}$ is the Green function (32) with $\bar{\Delta}=0$. Apart from an irrelevant constant, this leads to the expression for the effective action

$$
\begin{aligned}
S_{\mathrm{eff}} & =-i \operatorname{Tr} \ln \left[1-G_{0}\left(\begin{array}{cc}
0 & \Delta \\
\Delta^{*} & 0
\end{array}\right)\right] \\
& =i \operatorname{Tr} \sum_{\ell=1}^{\infty} \frac{1}{\ell}\left[G_{0}\left(\begin{array}{cc}
0 & \Delta \\
\Delta^{*} & 0
\end{array}\right)\right]^{\ell}=: \sum_{\ell=1}^{\infty} S_{\mathrm{eff}}^{(\ell)} .
\end{aligned}
$$

For $\ell=1$, the trace over the $2 \times 2$ matrix immediately yields zero. In a similar fashion all terms $S_{\text {eff }}^{(\ell)}$, with $\ell$ odd, give zero. For the quadratic term, we obtain

$$
S_{\mathrm{eff}}^{(2)}=i \operatorname{Tr} \frac{1}{p_{0}+\xi(\mathbf{p})} \Delta^{*} \frac{1}{p_{0}-\xi(\mathbf{p})} \Delta,
$$

where we recall the definition of the derivative $p_{\mu}$ as operating on everything that appears to the right. Applying the derivative expansion rules outlined above, we can cast the quadratic term in the effective action in the form

$$
S_{\mathrm{eff}}^{(2)}=i \operatorname{Tr} \frac{1}{k_{0}+\xi(\mathbf{k})} \frac{1}{k_{0}-i \partial_{0}-\xi(\mathbf{k}+i \nabla)} \Delta^{*} \Delta .
$$

Usually, the quartic terms are included in the Ginzburg-Landau theory without derivatives. We then can treat $p_{0} \pm \xi(\mathbf{p})$ as a c-number and

$$
S_{\text {eff }}^{(4)}=\frac{i}{2} \operatorname{Tr} \frac{1}{\left[k_{0}^{2}-\xi^{2}(\mathbf{k})\right]^{2}}|\Delta|^{4} .
$$

We may truncate the series in $(37)$ here, provided the pair field $\Delta$ is small as is the case in the vicinity of the phase transition.

To include the temperature $T$ in the theory, we adopt the imaginary-time approach to thermal field theory [18, 19]. Very briefly, it can be derived from the corresponding quantum field theory at zero temperature simply by going over to imaginary times, $t \rightarrow-i \tau$, and substituting

$$
\int \frac{\mathrm{d} k_{0}}{2 \pi} g\left(k_{0}\right) \rightarrow i \beta^{-1} \sum_{n} g\left(i \omega_{n}\right)
$$


where $g$ is an arbitrary function, while $\omega_{n}$ denote the Matsubara frequencies,

$$
\omega_{n}= \begin{cases}\pi \beta^{-1} 2 n, & \text { (bosonic) } \\ \pi \beta^{-1}(2 n+1), & \text { (fermionic) }\end{cases}
$$

with $n$ an integer and $\beta=1 / T$. With these rules, the Minkowski action $S$ goes over into

$$
S=\int_{x} \mathcal{L}(t, \mathbf{x}) \rightarrow-i \int_{0}^{\beta} \mathrm{d} \tau \int_{\mathbf{x}} \mathcal{L}(-i \tau, \mathbf{x})=: i S^{\mathrm{E}}
$$

where the superscript $\mathrm{E}$ on the action at the right-hand side is to indicate that it pertains to Euclidean rather than to Minkowski spacetime.

For the case at hand we obtain in this way the finite-temperature action:

$$
S_{\mathrm{eff}}^{\mathrm{E}}=\operatorname{Tr}\left[\frac{1}{i \omega_{n}+\xi(\mathbf{k})} \frac{1}{i \omega_{n}+\partial_{\tau}-\xi(\mathbf{k}+i \nabla)} \Delta^{*} \Delta+\frac{1}{2} \frac{1}{\left[\omega_{n}^{2}+\xi^{2}(\mathbf{k})\right]^{2}}|\Delta|^{4}\right],
$$

where the trace at finite temperature reads explicitly

$$
\operatorname{Tr}=\int_{0}^{\beta} \mathrm{d} \tau \int \mathrm{d}^{d} x \frac{\mathrm{d}^{d} k}{(2 \pi)^{d}} \beta^{-1} \sum_{n}
$$

Let us for the moment consider only the time-independent part of the effective action (44) and expand in gradients. The sums over the Matsubara frequencies are carried out with the help of the formulas

$$
\begin{aligned}
\beta^{-1} \sum_{n} \frac{1}{\omega_{n}^{2}+\xi^{2}} & =\frac{1}{2} \frac{X}{\xi} \\
\beta^{-1} \sum_{n} \frac{1}{\left(\omega_{n}^{2}+\xi^{2}\right)^{2}} & =-\frac{1}{4 \xi} \frac{\partial}{\partial \xi}\left(\frac{X}{\xi}\right) \\
\beta^{-1} \sum_{n} \frac{1}{i \omega_{n}+\xi} \frac{1}{\left(i \omega_{n}-\xi\right)^{2}} & =-\frac{1}{4} \frac{\partial}{\partial \xi}\left(\frac{X}{\xi}\right) \\
\beta^{-1} \sum_{n} \frac{1}{i \omega_{n}+\xi} \frac{1}{\left(i \omega_{n}-\xi\right)^{3}} & =-\frac{X}{8 \xi^{3}}-\frac{\beta}{16 \xi} \frac{\partial}{\partial \xi}\left(\frac{Y}{\xi}\right),
\end{aligned}
$$

where $X$ and $Y$ abbreviate the functions

$$
X=\tanh (\beta \xi / 2), \quad Y=1 / \cosh ^{2}(\beta \xi / 2) .
$$

As was first shown by Drechsler and Zwerger [10], most of the momentum integrals can be performed analytically for arbitrary values of the coupling constant in two space dimensions. In this way, they arrived at the time-independent Ginzburg-Landau theory describing the crossover from the weak-coupling BCS limit to the strong-coupling BEC limit:

$$
\mathcal{L}_{\mathrm{eff}}=c \Delta^{*} \nabla^{2} \Delta+a|\Delta|^{2}-\frac{1}{2} b|\Delta|^{4},
$$

with the coefficients

$$
\begin{gathered}
a=\frac{\nu(0 \AA}{2} \ln \left(\frac{4 \mathrm{e}^{\gamma}}{\pi}\right) \theta(\mu)+\ln \left(\beta \epsilon_{a} / 4\right)+\ln (\beta|\mu| / 2) \tanh (\beta \mu / 2) \\
\left.+\operatorname{sgn}(\mu) \int_{\beta|\mu| / 2}^{\infty} \mathrm{d} x \ln (x) Y(x)\right], \\
b=\frac{\nu(0)}{4}\left[\frac{7 \zeta(3)}{2 \pi^{2}} \beta^{2} \theta(\mu)-\frac{1}{\mu^{2}} \tanh (\beta \mu / 2)+\operatorname{sgn}(\mu) \frac{\beta^{2}}{4} \int_{\beta|\mu| / 2}^{\infty} \mathrm{d} x \frac{X(x)}{x^{3}}\right],
\end{gathered}
$$

and 


$$
c=\frac{\nu(0)}{8 m}\left[\frac{7 \zeta(3)}{2 \pi^{2}} \beta^{2} \mu \theta(\mu)+\frac{\beta^{2}}{4}|\mu| \int_{\beta|\mu| / 2}^{\infty} \mathrm{d} x \frac{X(x)}{x^{3}}\right] .
$$

Here, we introduced the integration variable $x=\beta \xi / 2, \gamma=0.577216 \cdots$ is Euler's constant, $\theta(x)$ is the Heaviside unit step function, $\operatorname{sgn}(x)$ the sign function, and $\zeta(x)$ Riemann's zeta function, with $\zeta(3)=1.20206 \cdots$. (The second term at the righthand side of Eq. (50) differs slightly from the corresponding term in Ref. [10], but is consistent with a later work by Zwerger and collaborator [20].)

When studying the time-dependence of the effective action (44), special care has to be taken with analytic continuation. We follow Sá de Melo, Randeria, and Engelbrecht [12] and first analytic continue, using the formula

$$
\beta^{-1} \sum_{n} \frac{1}{i \omega_{n}+\xi} \frac{1}{i \omega_{n}-i \omega_{l}-\xi}=-\left[\mathrm{P} \frac{1}{2 \xi+q_{0}}-i \pi \delta\left(2 \xi+q_{0}\right)\right] X,
$$

before expanding in time derivatives. (In Ref. [10], the expansion was done first, leading to results which are not consistent with those known in the BCS limit [21,22].) In Eq. (52), P stands for the principal part, while $\omega_{l}$ is a bosonic Matsubara frequency, which at the right-hand side is analytic continued to the real axes by replacing $i \omega_{l}$ with $q_{0}+i \eta$. In this way, we find for the dynamic part (in Minkowski spacetime)

$$
\mathcal{L}_{\mathrm{dyn}}=\left[Q^{\prime}\left(i \partial_{0}\right)-i \pi Q^{\prime \prime}\left(i \partial_{0}\right)\right] \Delta^{*} \Delta
$$

with

$$
\begin{aligned}
Q^{\prime}\left(q_{0}\right) & =-\mathrm{P} \int_{\mathbf{k}} \frac{q_{0}}{2 \xi\left(2 \xi+q_{0}\right)} X \\
Q^{\prime \prime}\left(q_{0}\right) & =\frac{1}{2} \nu(0) \tanh \left(\beta q_{0} / 4\right) \theta\left(\mu-q_{0} / 2\right) .
\end{aligned}
$$

The most important result to be noted here is that the low-energy dynamics is dissipative when the chemical potential $\mu$ is positive [12]. This is because the pairs can break up and decay into a continuum of fermionic excitations. On the other hand, for negative values of $\mu$, where the pairs are more tightly bound, the time-dependent Ginzburg-Landau theory describes a purely propagating pair mode. To investigate this point further, let us consider the two limits in detail.

\section{BCS limit}

In the weak-coupling BCS limit, where the chemical potential is well approximated by the Fermi energy $\mu=k_{\mathrm{F}}^{2} / 2 m$, with $k_{\mathrm{F}}$ the Fermi momentum, and $\epsilon_{a} / \epsilon_{\mathrm{F}} \rightarrow 0$, we recover the standard result (adjusted for the reduced space dimensionality) [22]

$$
\mathcal{L}_{\text {eff }}=\nu(0)\left\{\Delta^{*}\left[\ln \left(\frac{T_{0}}{T}\right)-\frac{\pi}{8 T_{0}} \partial_{0}+\frac{3 \xi_{0}^{2}}{v_{\mathrm{F}}^{2}}\left(\partial_{0}^{2}+\frac{v_{\mathrm{F}}^{2}}{2} \nabla^{2}\right)\right] \Delta-\frac{3 \xi_{0}^{2}}{v_{\mathrm{F}}^{2}}|\Delta|^{4}\right\}
$$

with $v_{\mathrm{F}}=k_{\mathrm{F}} / m$ the Fermi velocity, $\xi_{0}$ the BCS correlation length

$$
\xi_{0}^{2}=\frac{7 \zeta(3)}{48 \pi^{2}} \frac{v_{\mathrm{F}}^{2}}{T_{0}^{2}}
$$

and $T_{0}$ the BCS transition temperature [10]

$$
T_{0}=\frac{\mathrm{e}^{\gamma}}{\pi}\left(2 \mu \epsilon_{a}\right)^{1 / 2}
$$

expressed in terms of the binding energy $\epsilon_{a}$. Comparing the two terms in Eq. (55) involving time derivatives, we recognize a series expansion in powers of $q_{0} / T$; the terms without derivatives constitute an expansion in powers of $|\Delta| / T$ [23]. It therefore follows that Eq. (55) represents the first terms in a high-temperature expansion. Since we neglected time derivatives in the quartic and higher order terms, we are implicitly assuming that $q_{0}>>|\Delta|$ [22]. The time-dependent Ginzburg-Landau theory (55) obtained in the weak-coupling BCS limit is of the dissipative type often used in the context of defect formation in a symmetry-breaking phase transition (for reviews see Ref. [3] ]). For applications in the context of superconductivity see, for example, Refs. 24 26]. 


\section{E. BEC Limit}

In the strong-coupling BEC limit, the general form of the effective action (48) and (53) reduces to [10]

$$
\mathcal{L}_{\text {eff }}=\hat{\Delta}^{*}\left(i \partial_{0}+\mu_{\mathrm{b}}+\frac{\nabla^{2}}{4 m}\right) \hat{\Delta}-\lambda_{\mathrm{b}}|\hat{\Delta}|^{4},
$$

where we introduced a rescaled pair field

$$
\hat{\Delta}=\left(\frac{\nu(0)}{4|\mu|}\right)^{1 / 2} \Delta .
$$

The effective theory (58) is precisely of the form of a Gross-Pitaevski theory [27], describing a weakly interacting composite Bose gas with a mass $2 m$ (as expected), a small chemical potential which vanishes on approaching the critical temperature from below [see Eq. (27)]

$$
\mu_{\mathrm{b}}=2|\mu| \ln \left(\frac{\epsilon_{a}}{2|\mu|}\right)
$$

or using Eq. (26),

$$
\mu_{\mathrm{b}}=\frac{|\bar{\Delta}|^{2}}{\epsilon_{a}}
$$

and a repulsive contact interaction

$$
\lambda_{\mathrm{b}}=\frac{1}{\nu(0)}
$$

independent of the binding energy $\epsilon_{a}$ characterizing the interaction between the electrons. This is special to two dimensions; in $d$ space dimensions we have instead [28]

$$
\lambda_{\mathrm{b}}=(4 \pi)^{d / 2} \frac{1-d / 4}{\Gamma(2-d / 2)} \frac{\epsilon_{a}^{1-d / 2}}{m^{d / 2}},
$$

with $\Gamma(x)$ the Gamma function. Note the absence of any temperature-dependence in the effective theory (58). At zero temperature, exactly the same effective theory was obtained by Hausmann, using a self-consistent Green function method [11]. (See Ref. [28] for a derivation along the lines presented here.) This is, as that author argued, because in the BEC limit, the critical temperature $T_{0}$ is much smaller than the dissociation temperature $T_{\text {diss }} \approx \epsilon_{a}$ at which the tightly bound fermion pairs are broken up by thermal fluctuations. Hence, for all temperatures in the range $T \leq T_{0}<<T_{\text {diss }}$ we are effectively in the zero-temperature regime [11].

The Gross-Pitaevski theory (58) describes a gapless, purely propagating mode, viz. the Goldstone mode associated with the spontaneously broken global U(1) symmetry-the so-called Anderson-Bogoliubov mode [23].

The spacetime-dependent effective theory of a superconductor can only be derived in a few special cases [22]: one being close to the transition temperature where the expansion parameter is $1 / T$ and another being close to the absolute zero of temperature where the expansion parameter is $1 /|\bar{\Delta}|$. Outside these regimes, the effective theory depends on the ratio $|\nabla| / \partial_{0}$, and an expansion in both time derivatives and gradients is not possible. It is amusing to note that in going from the weak-coupling BCS limit to the strong-coupling BEC limit, we move from one valid regime to the other.

\section{DUAL THEORY}

In this section we investigate the dual formulation of the time-independent Ginzburg-Landau theory. This approach, in which the magnetic vortices of a superconductor play the central role, originates from lattice studies that started more than two decades ago [29 36]. These were in turn instigated by the success of the Kosterlitz-Thouless theory describing the phase transition in a superfluid film as the unbinding of vortex-antivortex pairs [1],2]. In the dual formulation of the Ginzburg-Landau theory, the superconductor-to-normal phase transition is understood as a proliferation of magnetic vortices. A detailed presentation of these matters as well as an extensive list of references to the literature can be found in Ref. [37]. 


\section{A. Electric Current Loops}

To account for the magnetic interaction we couple the Ginzburg-Landau theory in the usual, minimal way to a vector potential A. We also rescale the pair field such that $\beta$ times the Hamiltonian becomes

$$
\mathcal{H}_{\mathrm{GL}}=|(\nabla-2 i e \mathbf{A}) \phi|^{2}+m_{\phi}^{2}|\phi|^{2}+\lambda|\phi|^{4}+\frac{1}{2}(\nabla \times \mathbf{A})^{2}+\frac{1}{2 \alpha}(\nabla \cdot \mathbf{A})^{2},
$$

where we added a gauge-fixing term with parameter $\alpha$. To acquire a physical understanding of what this Hamiltonian describes [38], we recall that a $|\phi|^{4}$-theory gives a field theoretic description of strings with contact repulsion [39]. This equivalence rests on Feynman's observation [40] that the Green function

$$
G(\mathbf{x})=\int_{\mathbf{k}} \frac{\mathrm{e}^{i \mathbf{k} \cdot \mathbf{x}}}{\mathbf{k}^{2}+m_{\phi}^{2}}
$$

can be expressed as a path integral. An easy way to see this is to invoke Schwinger's proper-time method, which is based on Euler's form

$$
\frac{1}{a^{z}}=\frac{1}{\Gamma(z)} \int_{0}^{\infty} \frac{\mathrm{d} \tau}{\tau} \tau^{z} \mathrm{e}^{-\tau a}
$$

to write the right-hand side of (65) as [41]:

$$
\begin{aligned}
G(\mathbf{x}) & =\int_{0}^{\infty} \mathrm{d} \tau \mathrm{e}^{-\tau m_{\phi}^{2}} \int_{\mathrm{k}} \mathrm{e}^{i \mathbf{k} \cdot \mathbf{x}} \mathrm{e}^{-\tau \mathbf{k}^{2}} \\
& =\int_{0}^{\infty} \mathrm{d} \tau \mathrm{e}^{-\tau m_{\phi}^{2}}\left(\frac{1}{4 \pi \tau}\right)^{3 / 2} \mathrm{e}^{-\frac{1}{4} \mathbf{x}^{2} / \tau} .
\end{aligned}
$$

The factor $(1 / 4 \pi \tau)^{3 / 2} \exp \left(-\frac{1}{4} \mathbf{x}^{2} / \tau\right)$ appearing here can be interpreted as describing a Brownian string trajectory, showing that one endpoint of the string (located at $\mathbf{x}$ ) has a Gaussian distribution with respect to its other endpoint (located at the origin) [42]. If we imagine the string to be composed of $N$ links, each of length $a$, then in the limit where $N \rightarrow \infty, a \rightarrow 0$, the variable $\tau$ parameterizing the string stands for $\tau=N a^{2} / 6$. The integration over $\tau$ in (67) indicates that the string can be arbitrary long, but the weighing factor $\exp \left(-m_{\phi}^{2} \tau\right)$ exponentially suppresses long ones in the normal phase where $m_{\phi}^{2}>0$. To understand the physical meaning of this factor, let us return to the discrete string model and write

$$
m_{\phi}^{2}=6\left[\sigma(a)-\sigma_{\mathrm{cr}}\right] / a
$$

so that it becomes

$$
\mathrm{e}^{-m_{\phi}^{2} \tau}=\mathrm{e}^{-\sigma_{\text {eff }}(a) L}
$$

with $\sigma_{\text {eff }}(a)=\sigma(a)-\sigma_{\text {cr }}$ the effective string tension, and $L=N a$ the length of the string. The continuum limit is obtained by simultaneously letting $a \rightarrow 0$ and $\sigma(a) \rightarrow \sigma_{\mathrm{cr}}$, in such a way that the right-hand side of 68 tends to the finite value $m_{\phi}^{2}$. We thus see that the factor $\exp \left(-m_{\phi}^{2} \tau\right)$ weighs strings according to their lengths.

Following Feynman [40], we write the right-hand side of (67) as a path-integral, i.e., as a sum over all possible string trajectories having one endpoint at $\mathbf{x}(0)=0$ and the other at $\mathbf{x}(\tau)=\mathbf{x}$ [43]:

$$
G(\mathbf{x})=\int_{0}^{\infty} \mathrm{d} \tau \int_{\mathbf{x}(0)=0}^{\mathbf{x}(\tau)=\mathbf{x}} \mathrm{Dx}\left(\tau^{\prime}\right) \mathrm{e}^{-S_{0}}
$$

with the (Euclidean) action

$$
S_{0}=\int_{0}^{\tau} \mathrm{d} \tau^{\prime}\left[\frac{1}{4} \dot{\mathbf{x}}^{2}\left(\tau^{\prime}\right)+m_{\phi}^{2}\right]
$$

where $\dot{\mathbf{x}}(\tau)=\mathrm{d} \mathbf{x}(\tau) / \mathrm{d} \tau$.

In a similar way, also the partition function of the free theory, which written as a functional integral reads

$$
Z_{0}=\int \mathrm{D} \phi^{*} \mathrm{D} \phi \exp \left(-\int_{\mathbf{x}} \mathcal{H}_{0}\right)
$$


can be be represented as a path integral, this time involving only closed strings:

$$
\begin{aligned}
\ln \left(Z_{0}\right) & =-\ln \left[\operatorname{Det}\left(\mathbf{p}^{2}+m_{\phi}^{2}\right)\right]=-\operatorname{Tr} \ln \left(\mathbf{p}^{2}+m_{\phi}^{2}\right) \\
& =\int_{0}^{\infty} \frac{d \tau}{\tau} \mathrm{e}^{-\tau m_{\phi}^{2}} \int_{\mathbf{k}} \mathrm{e}^{-\tau \mathbf{k}^{2}}=\int_{0}^{\infty} \frac{d \tau}{\tau} \oint \operatorname{Dx}\left(\tau^{\prime}\right) \mathrm{e}^{-S_{0}} .
\end{aligned}
$$

Here, we used Euler's form (66) in the limit of small $z$. An additional factor $1 / \tau$ arises because one can start traversing a closed string anywhere along the loop. The $|\phi|^{4}$-interaction in the Ginzburg-Landau model can be shown to result in the additional term [4]

$$
S_{\lambda}=-\lambda \int_{0}^{\tau} \mathrm{d} \tau_{l}^{\prime} \mathrm{d} \tau_{k}^{\prime} \delta\left[\mathbf{x}\left(\tau_{l}^{\prime}\right)-\mathbf{x}\left(\tau_{k}^{\prime}\right)\right]
$$

in the action, which gives an extra weight each time two strings—one parameterized by $\tau_{l}^{\prime}$ and one by $\tau_{k}^{\prime}$ —intersect. Physically, it represents a repulsive contact interaction between strings. Finally, the coupling of the field $\phi$ to the magnetic vector potential A via the electric current

$$
\mathbf{j}_{e}=-2 e i\left(\phi^{*} \nabla \phi-\phi \nabla \phi^{*}\right)-2(2 e)^{2} \mathbf{A}|\phi|^{2}
$$

with a charge $2 e$, results in the extra term [37]

$$
S_{e}=2 i e \int_{0}^{\tau} \mathrm{d} \tau^{\prime} \dot{\mathbf{x}}\left(\tau^{\prime}\right) \cdot \mathbf{A}\left[\mathbf{x}\left(\tau^{\prime}\right)\right]
$$

showing that the strings described by the Ginzburg-Landau theory carry an electric current. Pasting the pieces together, we conclude that the partition function of the Ginzburg-Landau theory can be equivalently represented as a grand canonical ensemble of fluctuating electric current loops, of arbitrary length and shape [45]:

$$
Z=\int \mathrm{DA} \mathrm{e}^{-\frac{1}{2} \int_{\mathbf{x}}\left[(\nabla \times \mathbf{A})^{2}+\frac{1}{\alpha}(\nabla \cdot \mathbf{A})^{2}\right]} \sum_{N=0}^{\infty} \frac{1}{N !} \prod_{l=1}^{N}\left[\int_{0}^{\infty} \frac{\mathrm{d} \tau_{l}}{\tau_{l}} \oint \mathrm{Dx}\left(\tau_{l}^{\prime}\right)\right] \mathrm{e}^{-S_{\mathrm{GL}}}
$$

with the action

$$
\begin{aligned}
S_{\mathrm{GL}}= & \sum_{l=1}^{N} \int_{0}^{\tau_{l}} \mathrm{~d} \tau_{l}^{\prime}\left\{\frac{1}{4} \dot{\mathbf{x}}^{2}\left(\tau_{l}^{\prime}\right)+m_{\phi}^{2}+2 i e \dot{\mathbf{x}}\left(\tau_{l}^{\prime}\right) \cdot \mathbf{A}\left[\mathbf{x}\left(\tau_{l}^{\prime}\right)\right]\right\} \\
& +\lambda \sum_{l, k=1}^{N} \int_{0}^{\tau_{l}} \mathrm{~d} \tau_{l}^{\prime} \int_{0}^{\tau_{k}} \mathrm{~d} \tau_{k}^{\prime} \delta\left[\mathbf{x}\left(\tau_{l}^{\prime}\right)-\mathbf{x}\left(\tau_{k}^{\prime}\right)\right]
\end{aligned}
$$

On entering the superconducting phase, characterized by a sign change in the mass term of the Ginzburg-Landau theory, the effective string tension approaches zero as

$$
\sigma_{\text {eff }}(a) \propto m_{\phi}^{2} \propto\left(T-T_{\mathrm{c}}\right)^{2 \nu}
$$

with $\nu$ the correlation length exponent, and the electric current loops proliferate. This proliferation is also signaled by the absolute value $|\phi|$ of the pair field which then develops a vacuum expectation value, identifying it as the order parameter.

\section{B. Disorder Parameter}

The dual formulation of the Ginzburg-Landau gives a similar representation of the partition function as the path integral (77); however this time not in terms of electric current, but of magnetic vortex loops. To derive it, we start with a trick [46] and introduce a (hypothetical) magnetic monopole at some point $\mathbf{z}$ inside the superconductor. A monopole is a source of magnetic flux. Due to the Meissner effect, the flux lines emanating from the monopole are squeezed into a flux tube. In this way, we managed to create a magnetic vortex at zero external field. Electrodynamics in the presence of a monopole was first described by Dirac [47] who argued that the physical local magnetic induction $\mathbf{h}$ is given by the combination $\nabla \times \mathbf{A}(\mathbf{x})-\mathbf{B}^{\mathrm{P}}(\mathbf{x})$. The subtracted plastic field 


$$
B_{i}^{\mathrm{P}}(\mathbf{x})=\Phi_{0} \int_{L_{\mathbf{z}}} \mathrm{d} y_{i} \delta(\mathbf{x}-\mathbf{y}),
$$

with $\Phi_{0}=\pi / e$ the magnetic flux quantum, removes the field of the so-called Dirac string running along some path $L_{\mathbf{z}}$ from the location $\mathbf{z}$ of the monopole to infinity. On account of Stokes' theorem, the plastic field satisfies the equation

$$
\nabla \cdot \mathbf{B}^{\mathrm{P}}(\mathbf{x})=-\rho_{\mathrm{m}}(\mathbf{x})
$$

with $\rho_{\mathrm{m}}(\mathbf{x})=\Phi_{0} \delta(\mathbf{x}-\mathbf{z})$ the monopole density.

We continue by writing $\phi(\mathbf{x})$ as

$$
\phi(\mathbf{x})=\bar{\phi} \mathrm{e}^{2 i \varphi(\mathbf{x})}
$$

where $\bar{\phi}$ is a constant solution of the mean-field equation. This approximation, where the phase of the order field is allowed to vary in space while the modulus is kept fixed, is called the London limit. The Ginzburg-Landau Hamiltonian then becomes after integrating out the phase field $\varphi$ :

$$
\mathcal{H}_{\mathrm{GL}}^{\mathrm{P}}=\frac{1}{2}\left(\nabla \times \mathbf{A}-\mathbf{B}^{\mathrm{P}}\right)^{2}+\frac{1}{2} m_{A}^{2} A_{i}\left(\delta_{i j}-\frac{\partial_{i} \partial_{j}}{\nabla^{2}}\right) A_{j}+\frac{1}{2 \alpha}(\nabla \cdot \mathbf{A})^{2},
$$

where

$$
m_{A}=2 e|\bar{\phi}|
$$

is the inverse magnetic penetration depth $m_{A}=\lambda_{\mathrm{L}}^{-1}$. We gave $\mathcal{H}$ the superscript $\mathrm{P}$ to indicate the presence of the monopole. While the Dirac string is immaterial in the normal phase, Nambu [48] argued that it acquires physical relevance in the superconducting phase where it serves as the core of the magnetic vortex or Abrikosov flux tube originating at the monopole.

The energy $E_{V}$ of this configuration is easily obtained by substituting the solution of the field equation for the gauge field

$$
A_{i}(\mathbf{x})=\int_{\mathbf{y}} G_{i j}(\mathbf{x}-\mathbf{y})\left[\nabla \times \mathbf{B}^{\mathrm{P}}(\mathbf{y})\right]_{j},
$$

with $G_{i j}$ the gauge-field Green function

$$
G i j(\mathbf{x})=\int_{\mathbf{k}}\left(\frac{\delta_{i j}-k_{i} k_{j} / \mathbf{k}^{2}}{\mathbf{k}^{2}+m_{A}^{2}}+\alpha \frac{k_{i} k_{j}}{\mathbf{k}^{4}}\right) \mathrm{e}^{i \mathbf{k} \cdot \mathbf{x}},
$$

back into the Hamiltonian (33). The energy is divergent in the ultraviolet because in the London limit, where the mass $\left|m_{\phi}\right|$ of the $\phi$-field is taken to be infinite, the vortices are considered to be ideal lines. For a finite mass, a vortex core has a typical width of the order of the coherence length $\xi=1 /\left|m_{\phi}\right|$. This mass therefore provides a natural ultraviolet cutoff to the theory. Omitting the irrelevant (diverging) monopole self-interaction, one finds [48]

$$
E_{V}=\frac{1}{2} g^{2} \int_{L_{\mathbf{z}}} \mathrm{d} x_{i} \int_{L_{\mathbf{z}}} \mathrm{d} y_{i} G(\mathbf{x}-\mathbf{y})=\sigma_{V} L_{\mathbf{z}} .
$$

Here,

$$
G(\mathbf{x})=\int_{\mathbf{k}} \frac{\mathrm{e}^{i \mathbf{k} \cdot \mathbf{x}}}{\mathbf{k}^{2}+m_{A}^{2}}=\frac{1}{4 \pi} \frac{\mathrm{e}^{-m_{A}|\mathbf{x}|}}{|\mathbf{x}|}
$$

is the scalar Green function, $g$ abbreviates the combination

$$
g=\Phi_{0} m_{A},
$$

while $L_{\mathbf{z}}$ denotes the (infinite) length of the Dirac string and

$$
\sigma_{V}=\frac{1}{8 \pi} g^{2} \ln \left(\frac{\left|m_{\phi}\right|^{2}}{m_{A}^{2}}\right)=\frac{1}{4 \pi} g^{2} \ln \left(\kappa_{\mathrm{GL}}\right)
$$

its line tension, first calculated by Abrikosov [49]. The value $\kappa_{\mathrm{GL}}=1 / \sqrt{2}$ of the Ginzburg-Landau parameter 


$$
\kappa_{\mathrm{GL}}=\frac{\left|m_{\phi}\right|}{m_{A}}=\frac{\lambda_{\mathrm{L}}}{\xi}, \quad \text { or } \quad \kappa_{\mathrm{GL}}^{2}=\frac{\lambda}{2 e^{2}}
$$

separates the type-II regime $\left(\kappa_{\mathrm{GL}}>1 / \sqrt{2}\right)$, where isolated vortices can exist, from the type-I regime $\left(\kappa_{\mathrm{GL}}<1 / \sqrt{2}\right)$, where a partial penetration of an external field is impossible. Remembering that $L_{\mathbf{z}}$ was the Dirac string, we see from (87) that in the superconducting phase it indeed becomes the core of the magnetic vortex originating at the monopole, as was first observed by Nambu [48].

The operator $V\left(L_{\mathbf{z}}\right)$ describing the monopole with its emerging flux tube [50,51] is easily constructed by noting that in the functional-integral approach, a given field configuration is weighted with a Boltzmann factor $\exp \left(-\int_{\mathbf{x}} \mathcal{H}_{\mathrm{GL}}^{\mathrm{P}}\right)$, with the Hamiltonian given by Eq. 83). From this we infer that the explicit form of the vortex operator is

$$
V\left(L_{\mathbf{z}}\right)=\exp \left\{\int_{\mathbf{x}}\left[(\nabla \times \mathbf{A}) \cdot \mathbf{B}^{\mathrm{P}}-\frac{1}{2}\left(\mathbf{B}^{\mathrm{P}}\right)^{2}\right]\right\} .
$$

We next demonstrate that this operator can be used to distinguish the superconducting from the normal phase [50,51]. To this end, let us consider the correlation function $\left\langle V\left(L_{\mathbf{z}}\right) V^{*}\left(L_{\overline{\mathbf{z}}}\right)\right\rangle$, where $V^{*}\left(L_{\overline{\mathbf{z}}}\right)$ describes an additional antimonopole brought into the system at $\overline{\mathbf{z}}$, with $L_{\overline{\mathbf{z}}}$ being the accompanying Dirac string running from infinity to $\overline{\mathbf{z}}$. Since all the integrals involved are Gaussian, this expectation value can be evaluated directly. We proceed, however, in an indirect way for reasons that will become clear when we proceed, and first linearize the functional integral over the gauge field by introducing an auxiliary field $\tilde{\mathbf{h}}$. In the gauge $\nabla \cdot \mathbf{A}=0$, which corresponds to setting $\alpha=0$ in the Hamiltonian (83), we find [52]

$$
\begin{aligned}
& \left\langle V\left(L_{\mathbf{z}}\right) V^{*}\left(L_{\overline{\mathbf{z}}}\right)\right\rangle= \\
& \int \mathrm{DAD} \tilde{\mathbf{h}} \exp \left\{\int_{\mathbf{x}}\left[-\frac{1}{2} \tilde{\mathbf{h}}^{2}+i \tilde{\mathbf{h}} \cdot\left(\nabla \times \mathbf{A}-\mathbf{B}^{\mathrm{P}}\right)-\frac{m_{A}^{2}}{2} \mathbf{A}^{2}\right]\right\},
\end{aligned}
$$

where the plastic field satisfies (81) with the monopole density given by

$$
\rho_{\mathrm{m}}(\mathbf{x})=\Phi_{0}[\delta(\mathbf{x}-\mathbf{z})-\delta(\mathbf{x}-\overline{\mathbf{z}})]
$$

To appreciate the physical relevance of the auxiliary field, let us consider its field equation

$$
\tilde{\mathbf{h}}=i\left(\nabla \times \mathbf{A}-\mathbf{B}^{\mathrm{P}}\right)=i \mathbf{h} .
$$

It tells us that apart from a factor $i$, the auxiliary field $\tilde{\mathbf{h}}$ can be thought of as representing the local magnetic induction $\mathbf{h}$.

The integral over the vector potential is easily carried out by substituting the field equation for $\mathbf{A}$,

$$
\mathbf{A}=\frac{i}{m_{A}^{2}} \nabla \times \tilde{\mathbf{h}}
$$

back into (93), with the result

$$
\left\langle V\left(L_{\mathbf{z}}\right) V^{*}\left(L_{\overline{\mathbf{z}}}\right)\right\rangle=\int \mathrm{D} \tilde{\mathbf{h}} \exp \left\{-\frac{1}{2} \int_{\mathbf{x}}\left[\frac{1}{m_{A}^{2}}(\nabla \times \tilde{\mathbf{h}})^{2}+\tilde{\mathbf{h}}^{2}\right]-i \int_{\mathbf{x}} \tilde{\mathbf{h}} \cdot \mathbf{B}^{\mathrm{P}}\right\} .
$$

This shows that the magnetic vortex described by the plastic field $\mathbf{B}^{\mathrm{P}}$ couples to the fluctuating massive vector field $\tilde{\mathbf{h}}$, with a coupling constant $g$ introduced in Eq. (89). As the temperature approaches the critical temperature from below, $\bar{\phi}$ tends to zero, so that the vortex decouples from the auxiliary field $\tilde{\mathbf{h}}$. The finite magnetic penetration depth in the superconducting phase is reflected by the mass term in (97).

After carrying out the integral over $\tilde{\mathbf{h}}$ in 97 , we obtain for the correlation function

$$
\begin{aligned}
& \left\langle V\left(L_{\mathbf{z}}\right) V^{*}\left(L_{\overline{\mathbf{z}}}\right)\right\rangle= \\
& \exp \left\{-\frac{1}{2} \int_{\mathbf{x}, \mathbf{y}}\left[\rho_{\mathrm{m}}(\mathbf{x}) G(\mathbf{x}-\mathbf{y}) \rho_{\mathrm{m}}(\mathbf{y})+m_{A}^{2} B_{i}^{\mathrm{P}}(\mathbf{x}) G(\mathbf{x}-\mathbf{y}) B_{i}^{\mathrm{P}}(\mathbf{y})\right]\right\} .
\end{aligned}
$$

The first term in the argument of the exponential function contains a diverging monopole self-interaction for $\mathbf{x}=\mathbf{y}$. This divergence is irrelevant and can be eliminated by defining a renormalized operator

$$
V_{\mathrm{r}}\left(L_{\mathbf{z}}\right)=V\left(L_{\mathbf{z}}\right) \exp \left[\frac{1}{2} \Phi_{0}^{2} G(0)\right] .
$$

The second term in the argument is the most important one for our purposes. It represents a Biot-Savart interaction between two line elements $\mathrm{d} x_{i}$ and $\mathrm{d} y_{i}$ of the magnetic vortex (see Fig. 11). 


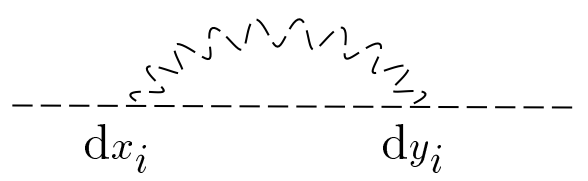

FIG. 1. Biot-Savart interaction (wiggly line) between two line elements $\mathrm{d} x_{i}$ and $\mathrm{d} y_{i}$ of a magnetic vortex (straight line).

For the renormalized operators we find

$$
\left\langle V_{\mathrm{r}}\left(L_{\mathbf{z}}\right) V_{\mathrm{r}}^{*}\left(L_{\overline{\mathbf{z}}}\right)\right\rangle=\exp \left(-\sigma_{V} L_{\mathbf{z} \overline{\mathbf{z}}}\right) \exp \left(\frac{\Phi_{0}^{2}}{4 \pi} \frac{\mathrm{e}^{-m_{A} L_{\mathbf{z} \overline{\mathbf{z}}}}}{L_{\mathbf{z} \overline{\mathbf{z}}}}\right),
$$

where $L_{\mathbf{z} \overline{\mathbf{z}}}$ denotes the length of the flux tube connecting the monopole at $\mathbf{z}$ with the antimonopole at $\overline{\mathbf{z}}$. Initially, the two Dirac strings may run to infinity along two arbitrary paths. Due to the string tension, however, they join on the shortest path $L_{\mathbf{z} \overline{\mathbf{z}}}$ connecting the monopoles.

The correlation function (100) behaves differently in the two phases. In the superconducting phase, where $m_{A} \neq 0$, the first factor dominates. It represents the confining linear potential between the monopole and the antimonopole. As a result, the correlation function decays exponentially for distances larger than $1 / \sigma_{V}$ :

$$
\left\langle V_{\mathrm{r}}\left(L_{z \bar{z}}\right) V_{\mathrm{r}}^{*}\left(L_{z \bar{z}}\right)\right\rangle \rightarrow 0 .
$$

On the other hand, in the normal phase, where the gauge field is massless, the confinement factor in the correlation function (100) disappears, while the argument of the second exponential turns into a pure Coulomb potential. The correlation function remains, consequently, finite for large distances:

$$
\left\langle V_{\mathrm{r}}\left(L_{z \bar{z}}\right) V_{\mathrm{r}}^{*}\left(L_{z \bar{z}}\right)\right\rangle \rightarrow 1,
$$

thus indicating a proliferation of magnetic vortices in the normal phase. This demonstrates that the vortex operator can be used as an order parameter to distinguish the two phases. Since it develops a vacuum expectation value not in the superconducting, but in the normal phase it is referred to as a disorder parameter.

It is interesting to consider in detail the limit $T \uparrow T_{\mathrm{c}}$, where the coupling constant $g=\Phi_{0} m_{A}$ tends to zero and the magnetic vortex decouples from the massive vector field [52]. In Eq. [97), this limit yields the constraint $\nabla \times \tilde{\mathbf{h}}=0$ which can be solved by setting $\tilde{\mathbf{h}}=\nabla \gamma$. The correlation function then takes the simple form

$$
\left\langle V_{\mathrm{r}}(\mathbf{z}) V_{\mathrm{r}}^{*}(\overline{\mathbf{z}})\right\rangle=\int \mathrm{D} \gamma \exp \left[-\frac{1}{2} \int_{\mathbf{x}}(\nabla \gamma)^{2}+i \int_{\mathbf{x}} \gamma \rho_{\mathrm{m}}\right] .
$$

In the absence of monopoles, the theory reduces to that of a free gapless mode $\gamma$ that may be thought of as representing the magnetic scalar potential:

$$
\nabla \gamma=i \nabla \times \mathbf{A} .
$$

This follows from combining the physical interpretation of the vector field $\mathbf{h}(95)$ with the equation $\tilde{\mathbf{h}}=\nabla \gamma$.

The correlation function (103) can be put in the form

$$
\left\langle V_{\mathrm{r}}(\mathbf{z}) V_{\mathrm{r}}^{*}(\overline{\mathbf{z}})=\left\langle\mathrm{e}^{i \Phi_{0}[\gamma(\mathbf{z})-\gamma(\overline{\mathbf{z}})]}\right\rangle,\right.
$$

where the average at the right-hand side is taken with respect to the free scalar theory. This equation shows that in the normal phase $\left(T>T_{\mathrm{c}}\right)$, the Dirac string looses its physical relevance, the right-hand side depending only on the end points $\mathbf{z}$ and $\overline{\mathbf{z}}$, not on the path $L_{\mathbf{z} \overline{\mathbf{z}}}$ connecting these points. The notion of a magnetic vortex is of no relevance in this phase because the vortices proliferate and carry no energy. This is the reason for omitting any reference to vortex lines in the argument of $V$ in Eqs. (103) and (105).

\section{Magnetic Vortex Loops}

From the above results for a single vortex, we can now easily infer the dual formulation of the Ginzburg-Landau theory. In this formulation, the partition function is written as a grand canonical ensemble of fluctuating magnetic vortex loops, of arbitrary length and shape: 


$$
Z=\int \mathrm{D} \tilde{\mathbf{h}} \mathrm{e}^{-\frac{1}{2} \int_{\mathbf{x}}\left[(\nabla \times \tilde{\mathbf{h}})^{2} / m_{A}^{2}+\frac{1}{2} \tilde{\mathbf{h}}^{2}\right]} \sum_{N=0}^{\infty} \frac{1}{N !} \prod_{l=1}^{N}\left[\int_{0}^{\infty} \frac{\mathrm{d} \tau_{l}}{\tau_{l}} \oint \operatorname{Dx}\left(\tau_{l}^{\prime}\right)\right] \mathrm{e}^{-S_{\text {dual }}}
$$

with the dual action, [cf. Eq. (78)]

$$
\begin{aligned}
S_{\text {dual }}= & \sum_{l=1}^{N} \int_{0}^{\tau_{l}} \mathrm{~d} \tau_{l}^{\prime}\left\{\frac{1}{4} \dot{\mathbf{x}}^{2}\left(\tau_{l}^{\prime}\right)+m_{\psi}^{2}+i \Phi_{0} \dot{\mathbf{x}}\left(\tau_{l}^{\prime}\right) \cdot \tilde{\mathbf{h}}\left[\mathbf{x}\left(\tau_{l}^{\prime}\right)\right]\right\} \\
& +u \sum_{l, k=1}^{N} \int_{0}^{\tau_{l}} \mathrm{~d} \tau_{l}^{\prime} \int_{0}^{\tau_{k}} \mathrm{~d} \tau_{k}^{\prime} \delta\left[\mathbf{x}\left(\tau_{l}^{\prime}\right)-\mathbf{x}\left(\tau_{k}^{\prime}\right)\right] .
\end{aligned}
$$

We have included here a mass term $m_{\psi}^{2}$ representing the intrinsic vortex line tension [cf. Eq. (68)], and also a contact repulsion between the vortices parameterized by $u$.

The equivalent field representation of this dual theory reads [53,54, $37,55,52,46$,

$$
Z=\int \mathrm{D} \tilde{\mathbf{h}} \mathrm{D} \psi^{*} \mathrm{D} \psi \exp \left(-\int_{\mathbf{x}} \mathcal{H}_{\text {dual }}\right)
$$

with the Hamiltonian

$$
\mathcal{H}_{\text {dual }}=\frac{1}{2 m_{A}^{2}}(\nabla \times \tilde{\mathbf{h}})^{2}+\frac{1}{2} \tilde{\mathbf{h}}^{2}+\left|\left(\nabla-i \Phi_{0} \tilde{\mathbf{h}}\right) \psi\right|^{2}+m_{\psi}^{2}|\psi|^{2}+u|\psi|^{4},
$$

where the disorder field $\psi$ is minimally coupled to the massive vector field $\tilde{\mathbf{h}}$, representing the local magnetic induction. The dual formulation contains the same information as the original, Ginzburg-Landau formulation. For example, the vortex line tension 90 appears in the dual theory as a one-loop on-shell mass correction stemming from the graph depicted in Fig. 11 which we now interpret as a Feynman graph of the dual theory (109), with the straight and wiggly line denoting respectively the $\psi$ and $\tilde{\mathbf{h}}$-field Green function. Also the fixed points of the Ginzburg-Landau theory map onto those of the dual field theory [55]. And both formulations can be used to study the critical behavior of the superconductor-to-normal phase transition (see Refs. [30,34, 37,56,46, 57,58, for the dual approach).

\section{Acknowledgments}

It is a pleasure to thank N. Antunes, L. Bettencourt, A. Leggett, D. Steer, and G. Volovik for useful discussions during the NATO Winter School and European Science Foundation (ESF) Workshop Topological Defects and the Non-Equilibrium Dynamics of Symmetry Breaking Phase Transitions, Les Houches, February 16-26, 1999, and M. Crisan for helpful correspondence.

This work is performed as part of a scientific network supported by the ESF (see network's URL, http://www.physik.fuberlin.de/ $\sim$ defect).

[1] V. L. Berezinskii, Sov. Phys. JETP 34, 610 (1972).

[2] J. M. Kosterlitz and D. J. Thouless, J. Phys. C 6, 1181 (1973).

[3] NATO Advanced Study Institute Formation and Interactions of Topological Defects edited by A.-C. Davis and R. Brandenberger, (Plenum Press, New York, 1995)

[4] L. P. Gorkov, Sov. Phys. JETP 9, 1364 (1959).

[5] J. Bardeen, L. N. Cooper, J. R. Schrieffer, Phys. Rev. 108, 1175 (1957).

[6] V. L. Ginzburg and L. D. Landau, Zh. Eksp. Teor. Fiz. 20 (1950) 1064; reprinted in L. D. Landau, Collected Papers (Pergamon, London, 1965), p. 546.

[7] A. M. J. Schakel, On broken symmetries in Fermi systems, Ph. D. Thesis, University of Amsterdam (1989).

[8] D. M. Eagles, Phys. Rev. 186 (1969) 456.

[9] A. J. Leggett in Modern Trends in the Theory of Condensed Matter, edited by A. Pekalski and J. Przystawa (Springer-Verlag, Berlin, 1980), p. 13.

[10] M. Drechsler and W. Zwerger, Ann. Phys. (Germany) 1, 15 (1992).

[11] R. Haussmann, Z. Phys. B 91, 291 (1993).

[12] C. A. R. Sá de Melo, M. Randeria, and J. R. Engelbrecht, Phys. Rev. Lett. 71, 3202 (1993). 
[13] M. Marini, F. Pistolesi, and G. C. Strinati, Eur. Phys. J. 1, 151 (1998).

[14] V. N. Popov, Functional Integrals and Collective Excitations (Cambridge University Press, Cambridge, 1987).

[15] M. Randeria, J. -M. Duan, and L. -Y. Shieh, Phys. Rev. B 41, 327 (1990).

[16] R. D. Mattuck, A Guide to Feynman Diagrams in the Many-Body Problem (McGraw-Hill, New York, 1976).

[17] C. M. Fraser, Z. Phys. C 28, 101 (1985); I. J. R. Aitchison and C. M. Fraser, Phys. Rev. D 31, 2605 (1985).

[18] R. J. Rivers, Path Integrals in Quantum Field Theory (Cambridge University Press, Cambridge, 1987).

[19] J. I. Kapusta, Finite-Temperature Field Theory (Cambridge University Press, Cambridge, 1989).

[20] S. Stintzing and W. Zwerger, e-print cond-mat/9703129 (1997).

[21] A. Schmid, Phys. Kond. Materie 5, 302 (1966).

[22] E. Abrahams and T.Tsuneto, Phys. Rev. 152, 416 (1966).

[23] A. M. J. Schakel, Int. J. Mod. Phys. B 8, 2021 (1994).

[24] P.G. de Gennes, Superconductivity in Metals and Alloys (Benjamin, New York, 1966).

[25] M. Tinkham, Introduction to Superconductivity (McGraw-Hill, New York, 1975).

[26] M. Crisan, Theory of Superconductivity (World Scientific, Singapore, 1989).

[27] E. P. Gross, Nuovo Cimento 20, 454 (1961); L. P. Pitaevskii, Sov. Phys. JETP 13, 451 (1961).

[28] A. M. J. Schakel, in Correlations, Coherence, and Order, edited by D. V. Shopova and D. I. Uzunov (Plenum Press, New York, 1999), p. 295.

[29] T. Banks, B. Meyerson, and J. Kogut, Nucl. Phys. B 129, 493 (1977).

[30] M. Peskin, Ann. Phys. 113, 122 (1978).

[31] P. R. Thomas and M. Stone, Nucl. Phys. B 144, 513 (1978).

[32] W. Helfrich and W. Müller, in Continuum Models of Discrete Systems (Waterloo University Press, Waterloo, 1980), p. 753.

[33] C. Dasgupta and B. I. Halperin, Phys. Rev. Lett. 47, 1556 (1981).

[34] H. Kleinert, Lett. Nuovo Cimento 35, 405 (1982)

[35] J. Bartholomew, Phys. Rev. B 28 (1983) 5378.

[36] R. Savit, Rev. Mod. Phys. 52, 453 (1989).

[37] H. Kleinert, Gauge Fields in Condensed Matter (World Scientific, Singapore, 1989), Vol. I.

[38] A. M. J. Schakel, Boulevard of Broken Symmetries, e-print cond-mat/9805152 (1998).

[39] K. Symanzik, in Euclidean Quantum Field Theory, edited by R. Jost (Academic, New York, 1969).

[40] R. P. Feynman. Phys. Rev. 80, 440 (1950).

[41] J. Schwinger, Phys. Rev. 82, 664 (1951).

[42] H. Kleinert, Path Integrals in Quantum Mechanics, Statistics and Polymer Physics, 2nd Edition (World Scientific, Singapore, 1995).

[43] R. P. Feynman, Rev. Mod. Phys. 20, 367 (1948).

[44] G. Parisi, Statistical Field Theory (Addison-Wesley, New-York, 1988).

[45] E. Copeland, D. Haws, S. Holbraad, and R. Rivers in The Formation and Evolution of Cosmic Strings, edited by G. W. Gibbons, S. W. Hawking, and T. Vachaspati (Cambridge University Press, Cambridge, 1990), p. 35; ibid p. 49.

[46] M. Kiometzis, H. Kleinert, and A. M. J. Schakel, Fortschr. Phys. 43, 697 (1995).

[47] P. A. M. Dirac, Phys. Rev. 74, 817 (1948).

[48] Y. Nambu, Phys. Rev. D 10, 4262 (1974).

[49] A. A. Abrikosov, Sov. Phys. JETP 5, 1174 (1957).

[50] E. C. Marino, Phys. Rev. D 38, 3194 (1988); E. C. Marino, G. C. Marques, R. O. Ramos, and J. S. Ruiz, Phys. Rev. D 45, 3690 (1992); E. C. Marino, Ann. Phys. (NY) 224, 225 (1993).

[51] A. Kovner, B. Rosenstein, and D. Eliezer, Mod. Phys. Lett. A 5, 2733 (1990); Nucl Phys. B 350, 325 (1991); A. Kovner and B. Rosenstein, Phys. Rev. Lett. 67, 1490 (1991).

[52] M. Kiometzis and A. M. J. Schakel, Int. J. Mod. Phys. B 7, 4271 (1993).

[53] K. Bardakci and S. Samuel, Phys. Rev. D 18, 2849 (1978).

[54] H. Kawai, Prog. Theor. Phys. 65, 351 (1981).

[55] A. Kovner, P. Kurzepa, and B. Rosenstein, Mod. Phys. Lett. A 8, 1343 (1993).

[56] M. Kiometzis, H. Kleinert, and A. M. J. Schakel, Phys. Rev. Lett. 73, 1975 (1994).

[57] I. F. Herbut, J. Phys. A: Math. Gen. 29, 423 (1996).

[58] C. de Calan and F. S. Nogueira, e-print cond-mat/9903247(1999). 